\title{
Mobile Fitness and Weight Management Apps: Protocol for a Quality Evaluation
}

Madison Milne-Ives ${ }^{1}$, BAS, MSc; Ching Lam ${ }^{1}$, MEng; Michelle van Velthoven ${ }^{1}$, BSc, MSc; Edward Meinert ${ }^{1,2,3}$, MA, MSc, MBA, MPA, PhD

${ }_{1}^{1}$ Digitally Enabled PrevenTative Health Research Group, Department of Paediatrics, University of Oxford, Oxford, United Kingdom

${ }^{2}$ Department of Primary Care and Public Health, Imperial College London, London, United Kingdom

${ }^{3}$ Centre for Health Technology, Faculty of Health, University of Plymouth, Plymouth, United Kingdom

\section{Corresponding Author:}

Edward Meinert, MA, MSc, MBA, MPA, PhD

Centre for Health Technology

Faculty of Health

University of Plymouth

6 Kirkby Place

Plymouth, PL4 8AA

United Kingdom

Phone: 441752600600

Email: edward.meinert@plymouth.ac.uk

\begin{abstract}
Background: Obesity is a contributing factor for many noncommunicable diseases and a growing problem worldwide. Many mobile apps have been developed to help users improve their fitness and weight management behaviors. However, the speed at which apps are created and updated means that it is important to periodically assess their quality.

Objective: The purpose of this study is to evaluate the quality of fitness and weight management mobile health apps using the Mobile Application Rating Scale (MARS). It will also describe the features of the included apps and compare the results to a previous evaluation conducted in 2015.

Methods: Searches for "fitness," "weight," "exercise," "physical activity," "diet," "eat*," and "food" will be conducted in the Apple App Store and Google Play. Apps that have been updated over the past 5 years will be included. Two reviewers will rate the apps' quality using the MARS objective and subjective quality subscales. Interrater reliability will also be assessed. Features included in high-quality apps will be assessed, and changes in quality, features, and behavior change techniques made during the past 5 years will be described.
\end{abstract}

Results: The results will be included in the evaluation paper, which we aim to publish in 2020 .

Conclusions: This evaluation will assess the quality of currently available fitness and weight management apps.

International Registered Report Identifier (IRRID)： PRR1-10.2196/17685

(JMIR Res Protoc 2020;9(9):e17685) doi: 10.2196/17685

\section{KEYWORDS}

mobile apps; telemedicine; smartphone; exercise; weight loss; obesity; physical fitness; fitness trackers

\section{Introduction}

The number of people who are overweight or obese has tripled since 1975 , and in $2016,40 \%$ of adults were overweight [1]. Obesity is a major concern for public health because it increases the likelihood of many preventable diseases (eg, cardiovascular diseases, diabetes, osteoarthritis, and some cancers) and places an economic burden on the health care system $[1,2]$. However, obesity is largely a preventable condition [3]. Increasing physical activity and eating healthier foods can help people to manage their weight, and thus reduce the consequences of chronic and preventable diseases $[1,4]$.

Since the first smartphone was released in 2008, digital technologies have become an increasingly common and popular way for people to change their health behaviors [5]. Mobile apps are a useful platform to provide behavioral interventions 
to improve fitness and weight because of the widespread use of smartphones and the large number of mobile health apps available [6]. Some evidence of the acceptability and effectiveness of mobile health apps for increasing physical activity, improving eating behaviors, and reducing weight has been found [4,7], but additional evidence is still needed to strengthen the conclusion that mobile apps are effective at improving health outcomes in particular [8-10]. The mixed body of evidence of effectiveness might be due to the fact that higher engagement has been found to be related to increased adherence to the app and weight loss [4]. Therefore, it is important to assess the quality of mobile fitness and weight management apps because quality is likely to influence engagement, which will affect the effectiveness of these apps at changing behavior and causing weight loss.

Evaluations have previously examined the quality of mobile weight management apps [11,12]. However, testing for these evaluations was conducted in August 2014 [12] and the beginning of 2015 [11]. Given the rate at which apps are being developed and updated [13], evaluations should be conducted every couple of years to assess the quality of currently popular mobile fitness and weight management apps. Evaluations can then be compared to track whether there have been any changes in the quality or features of popular apps over time. Both of the previous evaluations assessed popular iOS and Android apps. Bardus et al [11] evaluated the apps using the MARS subscales and found that the overall quality of apps was moderate, while Chen et al [12] focused on Australian apps and concluded that the overall quality was suboptimal. The authors of both reviews also examined the behavior change techniques (BCTs) [14] that were incorporated in the apps. Experts have established a theory-based taxonomy of these BCTs to aid identification and evaluation of the key components of behavioral interventions [14]. The review of Australian apps found a general lack of BCTs [12], while self-monitoring of behavior and outcomes, goal setting for behavior and outcomes, and feedback on behavior and outcomes were identified as the most common BCTs in Bardus et al's review [11].

In their evaluation, Bardus et al [11] concluded that improvements could be made to app quality by focusing on information quality and evidence-based content. A similar, more current evaluation will provide an update on both the quality of mobile fitness and weight management apps and which BCTs are included in them. This will allow an assessment of whether and how mobile fitness and weight management apps have changed in the past 5 years. There are also improvements that can be made to the previous review methodologies: Bardus et al's evaluation [11] only included apps that focused on a combination of diet and physical activity interventions and had a version available in both the Apple App Store and Google Play, while Chen et al's review [12] did not use a standardized overall measure of app quality (the MARS measure was not yet published [15]). The proposed evaluation will broaden that scope by also including apps that focus on only diet or physical activity and apps available only in the App Store or Google Play, as this will better represent the broad range of apps that people use to improve their fitness and weight management. Additionally, our evaluation will compare our findings with
Bardus et al's [11] to examine how the general state of app quality and the inclusion of BCTs have changed in the past 5 years.

Therefore, this evaluation will be focused on three main research questions. First, what is the objective and subjective quality of various Apple and Android mobile fitness and weight management apps, as measured by the Mobile Application Rating Scale? Second, what are the features most commonly associated with high-quality apps? Third, how have the quality and included BCTs of popular apps changed since 2015?

\section{Methods}

\section{Overview}

We will use the PRISMA-P (Preferred Reporting Items for Systematic Reviews and Meta-Analyses Protocols) guidelines [16] to guide the search and selection of apps for evaluation. This evaluation will be composed of an app search, app selection, data extraction, data analysis, and data synthesis.

\section{Search Strategy}

We will search the Apple App Store and Google Play to identify current popular mobile fitness and weight management apps. We will search each of the following keywords: "fitness," "weight," "exercise," "physical activity," "diet," "eat*," and "food." These were chosen based on commonly used terms in the literature [4]. The search results will be filtered by popularity (based on the stores' display algorithms) and the 100 most popular apps from each search will be screened. This will ensure that the apps being evaluated are the ones that are most used and will limit the number of apps to be evaluated.

\section{Eligibility}

\section{Inclusion Criteria}

We will include popular apps that aim to improve health-related fitness and weight management behaviors, specifically diet or physical activity, and that target the general population. This will include apps designed for any age group, from children to older adults.

\section{Exclusion Criteria}

We will exclude duplicates (if an app is available for both iOS and Android operating systems, we will include the $\mathrm{iOS}$ version only) and apps that are not in English. We will also exclude apps that have not been updated in the past 5 years [17]. We will exclude any apps that do not provide dietary or physical activity behavioral interventions that aim to improve general health and fitness or weight management. Therefore, recipe apps, athletic training apps, and apps that are focused on behaviors that are not health-related (like looking younger) will be excluded. Apps that are focused on specific populations (eg, people with specific diseases or pregnant women) will also be excluded.

\section{Screening and App Selection}

All of the apps found in the search will be recorded in an Excel document (Microsoft Corp) and duplicates will be removed, including Android apps that also have an iOS version. 
Preliminary screening by two independent reviewers will determine initial eligibility for the evaluation using the information provided in the app summaries on the App Store and Google Play. Apps that are deemed eligible will be downloaded. Apps that, upon closer examination, do not meet the inclusion criteria will also be excluded. Any disagreements between the reviewers will be discussed and, if necessary, settled by a third reviewer. All of the apps identified as being eligible for inclusion will be reviewed. A PRISMA flow diagram will be used to record the details of the search, screening, and selection processes so that the evaluation can be reproduced.

\section{Data Extraction}

The apps will be tested and evaluated by two independent reviewers. Each app will be used for at least half an hour before being rated using the MARS scales $[15,18]$. The reviewers will also extract general information about the app as well as its features (eg, how it tracks behaviors or outcomes; if it provides notifications, feedback, or information) and any BCTs [14] that are included. The items to be extracted are summarized in Textbox 1.

Textbox 1. Data that will be extracted from the apps.

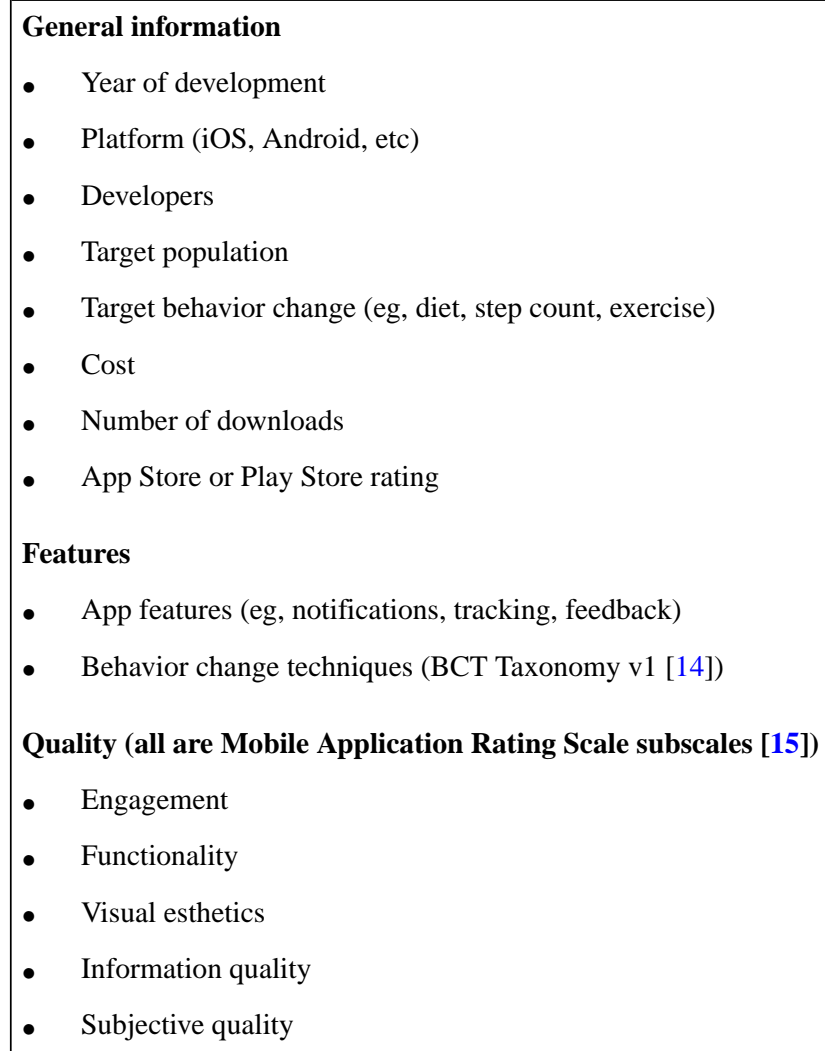

\section{Data Analysis and Synthesis}

The Mobile Application Rating Scale will be used to evaluate the quality of the included apps. Both reviewers will complete a training exercise in the MARS (which will be requested from the corresponding author of the MARS development paper) before conducting the evaluation [15]. The MARS has a total of 23 items split into 5 different subscales: engagement, functionality, esthetics, information, and subjective quality. Each item is rated on a 5-point Likert scale. The overall score is calculated by averaging the mean scores of the subscales, with objective and subjective ratings kept separate [15] Interrater reliability of the two reviewers will also be assessed.

The objective and subjective scores of the various apps will be compared to determine which apps have the highest quality. The features and BCTs of the 20 highest-rated apps will also be examined, to determine which features are associated with the highest-quality apps.

\section{Results}

The results will be included in the evaluation paper, which we aim to publish in 2020 .

\section{Discussion}

A systematic evaluation of mobile fitness and weight management apps will provide a clearer assessment of their quality. There are many fitness and weight management apps to choose from, and star rating systems have not been found to be strongly correlated with the MARS measure of app quality [15]. An evaluation of these apps will help consumers choose higher quality apps and will contribute to the literature on and the improvement of mobile health behavior change apps by examining which features and BCTs [14] are common in high-quality apps. These results will be compared to a previous evaluation of the quality of and BCTs included in mobile weight management apps and describe whether and how popular apps 
have changed since 2015 [11]. Based on the data, this section will compare the included apps, discuss the limitations of the evaluation, and consider important directions for future research. One limitation that can already be identified is the use of only two reviewers. Given the significant time requirements to evaluate each app in depth, it is only feasible to use two reviewers; although the reviewers will work independently, it is possible that they will be biased in a way that might not be identified.

\section{Acknowledgments}

CL, MMI, MHVV, and EM are supported by grants from EIT Health (grant 18654) and Promoting the Internet of Things via Collaborations between HEIS and Industry (PITCH-IN). EM is supported by the Sir David Cooksey Fellowship at the University of Oxford.

\section{Authors' Contributions}

EM, CL, and MMI conceived the study topic and designed the review protocol. MMI prepared the first draft of the protocol with revisions from MHVV and EM.

\section{Conflicts of Interest}

None declared.

\section{References}

1. World Health Organization. Obesity and Overweight. 2018. URL: https://www.who.int/en/news-room/fact-sheets/detail/ obesity-and-overweight [accessed 2020-08-03]

2. Fenton K. Health Matters: Obesity and the food environment. Public Health England Blogs. 2017. URL: https:/ /publichealthmatters.blog.gov.uk/2017/03/31/health-matters-obesity-and-the-food-environment/ [accessed 2020-08-03]

3. Hruby A, Hu FB. The Epidemiology of Obesity: A Big Picture. Pharmacoeconomics 2015 Jul;33(7):673-689 [FREE Full text] [doi: 10.1007/s40273-014-0243-x] [Medline: 25471927]

4. Dounavi K, Tsoumani O. Mobile Health Applications in Weight Management: A Systematic Literature Review. Am J Prev Med 2019 Jun;56(6):894-903 [FREE Full text] [doi: 10.1016/j.amepre.2018.12.005] [Medline: $\underline{31003801]}$

5. McKay FH, Cheng C, Wright A, Shill J, Stephens H, Uccellini M. Evaluating mobile phone applications for health behaviour change: A systematic review. J Telemed Telecare 2018 Jan;24(1):22-30. [doi: 10.1177/1357633X16673538] [Medline: 27760883]

6. Ferrara G, Kim J, Lin S, Hua J, Seto E. A Focused Review of Smartphone Diet-Tracking Apps: Usability, Functionality, Coherence With Behavior Change Theory, and Comparative Validity of Nutrient Intake and Energy Estimates. JMIR mHealth uHealth 2019 May 17;7(5):e9232 [FREE Full text] [doi: 10.2196/mhealth.9232] [Medline: $\underline{31102369]}$

7. Villinger K, Wahl DR, Boeing H, Schupp HT, Renner B. The effectiveness of app-based mobile interventions on nutrition behaviours and nutrition-related health outcomes: A systematic review and meta-analysis. Obes Rev 2019 Oct;20(10):1465-1484. [doi: 10.1111/obr.12903] [Medline: 31353783]

8. Milne-Ives M, Lam C, De Cock C, Van Velthoven MH, Meinert E. Mobile Apps for Health Behavior Change in Physical Activity, Diet, Drug and Alcohol Use, and Mental Health: Systematic Review. JMIR mHealth uHealth 2020 Mar 18;8(3):e17046 [FREE Full text] [doi: 10.2196/17046] [Medline: 32186518]

9. Ghelani DP, Moran LJ, Johnson C, Mousa A, Naderpoor N. Mobile Apps for Weight Management: A Review of the Latest Evidence to Inform Practice. Front Endocrinol (Lausanne) 2020;11:412 [FREE Full text] [doi: 10.3389/fendo.2020.00412] [Medline: 32670197]

10. Laing BY, Mangione CM, Tseng C, Leng M, Vaisberg E, Mahida M, et al. Effectiveness of a smartphone application for weight loss compared with usual care in overweight primary care patients: a randomized, controlled trial. Ann Intern Med 2014 Nov 18;161(10 Suppl):S5-12. [doi: 10.7326/M13-3005] [Medline: 25402403]

11. Bardus M, van Beurden SB, Smith JR, Abraham C. A review and content analysis of engagement, functionality, aesthetics, information quality, and change techniques in the most popular commercial apps for weight management. Int J Behav Nutr Phys Act 2016 Mar 10;13:35 [ FREE Full text] [doi: 10.1186/s12966-016-0359-9] [Medline: 26964880]

12. Chen J, Cade JE, Allman-Farinelli M. The Most Popular Smartphone Apps for Weight Loss: A Quality Assessment. JMIR mHealth uHealth 2015;3(4):e104 [FREE Full text] [doi: 10.2196/mhealth.4334] [Medline: 26678569]

13. Larson RS. A Path to Better-Quality mHealth Apps. JMIR mHealth uHealth 2018 Jul 30;6(7):e10414 [FREE Full text] [doi: 10.2196/10414] [Medline: $\underline{30061091]}$

14. Michie S, Richardson M, Johnston M, Abraham C, Francis J, Hardeman W, et al. The behavior change technique taxonomy (v1) of 93 hierarchically clustered techniques: building an international consensus for the reporting of behavior change interventions. Ann Behav Med 2013 Aug;46(1):81-95. [doi: 10.1007/s12160-013-9486-6] [Medline: 23512568]

15. Stoyanov SR, Hides L, Kavanagh DJ, Zelenko O, Tjondronegoro D, Mani M. Mobile app rating scale: a new tool for assessing the quality of health mobile apps. JMIR mHealth uHealth 2015;3(1):e27 [FREE Full text] [doi: 10.2196/mhealth.3422] [Medline: 25760773 ] 
16. Shamseer L, Moher D, Clarke M, Ghersi D, Liberati A, Petticrew M, PRISMA-P Group. Preferred reporting items for systematic review and meta-analysis protocols (PRISMA-P) 2015: elaboration and explanation. BMJ 2015 Jan 02;350:g7647 [FREE Full text] [doi: 10.1136/bmj.g7647] [Medline: 25555855]

17. Anderson K, Burford O, Emmerton L. App Chronic Disease Checklist: Protocol to Evaluate Mobile Apps for Chronic Disease Self-Management. JMIR Res Protoc 2016 Nov 04;5(4):e204 [FREE Full text] [doi: 10.2196/resprot.6194] [Medline: 27815233]

18. Mani M, Kavanagh DJ, Hides L, Stoyanov SR. Review and Evaluation of Mindfulness-Based iPhone Apps. JMIR mHealth uHealth 2015;3(3):e82 [FREE Full text] [doi: 10.2196/mhealth.4328] [Medline: 26290327]

\title{
Abbreviations \\ BCT: behavior change technique \\ MARS: Mobile Application Rating Scale \\ PRISMA-P: Preferred Reporting Items for Systematic Reviews and Meta-Analyses Protocols
}

\author{
Edited by $G$ Eysenbach; submitted 03.01.20; peer-reviewed by H Chen, $M$ Hong; comments to author 29.01.20; revised version \\ received 20.07.20; accepted 21.07.20; published 24.09.20 \\ Please cite as: \\ Milne-Ives M, Lam C, van Velthoven M, Meinert $E$ \\ Mobile Fitness and Weight Management Apps: Protocol for a Quality Evaluation \\ JMIR Res Protoc 2020;9(9):e17685 \\ URL: http://www.researchprotocols.org/2020/9/e17685/ \\ doi: $\underline{10.2196 / 17685}$ \\ PMID: $\underline{32969830}$
}

CMadison Milne-Ives, Ching Lam, Michelle van Velthoven, Edward Meinert. Originally published in JMIR Research Protocols (http://www.researchprotocols.org), 24.09.2020. This is an open-access article distributed under the terms of the Creative Commons Attribution License (https://creativecommons.org/licenses/by/4.0/), which permits unrestricted use, distribution, and reproduction in any medium, provided the original work, first published in JMIR Research Protocols, is properly cited. The complete bibliographic information, a link to the original publication on http://www.researchprotocols.org, as well as this copyright and license information must be included. 